\title{
Can the physical act of sexual intercourse before frozen embryo transfer improve IVF treatment outcome? A Randomized Clinical Trial
}

Jin-wei Hou

Shandong University of Traditional Chinese Medicine

Li-hua Yuan

The Affiliated Hospital of Shandong University of Traditional Chinese Medicine

Xian-ling Cao

Shandong University of Traditional Chinese Medicine

Jing-yan Song

The Affiliated Hospital of Shandong University of Traditional Chinese Medicine

Zhen-gao Sun ( $\nabla$ sunzhengao77@126.com )

The Affiliated Hospital of Shandong University of Traditional Chinese Medicine

\section{Research Article}

Keywords: Sexual intercourse, Barrier (condom) contraception, IVF, FET

Posted Date: October 5th, 2022

DOI: https://doi.org/10.21203/rs.3.rs-48030/v4

License: (c) (i) This work is licensed under a Creative Commons Attribution 4.0 International License. Read Full License 


\section{Abstract \\ Background}

Exposure of the female reproductive tract to either seminal plasma or fluid component of the ejaculate is beneficial to achieving successful embryo implantation and normal embryo development. But whether the "physical" component of of sexual intercourse during the peri-transfer period have any influence on FET pregnancy outcome is not clear.

\section{Methods}

We conducted a randomized trial that included 223 patients undergoing IVF treatment at a Universityaffiliated reproductive center from 19 July 2018 to 24 February 2019. Enrolled patients undergoing IVF treatment were randomized either to engage sexual intercourse using the barrier contraception (Group A, $n=116$ ) or to abstain (Group B, $n=107$ ) one night before FET. The follow-up parameters included biochemical losses rate, clinical pregnancy, early abortion and implantation rate.

\section{Results}

Patients having intercourse had higher clinical pregnancy rate $(51.72 \%$ vs. $37.07 \%, P=0.045)$ and implantation rate $(38.31 \%$ vs. $24.77 \%, P=0.005)$ compared to those did not engage intercourse. However, there was no significant difference of the spontaneous abortion rate between two groups $(11.67 \% 33$ vs. $14.63 \%, P=0.662)$.

\section{Conclusions}

Sexual intercourse before the day of embryo transfer may improve the clinical pregnancy and implantation rates during the frozen- thawed embryo transfer (FET) cycle. However, it should be noted that patients choose only one time for sexual intercourse, that is, the night before embryo transfer.

\section{TRIAL REGISTRATION NUMBER:}

This study was registered at the China Clinical Trial Registration Center, with the registration number ChiCTR1800017209.

\section{Background}

In the process of natural sexual reproduction, the act of intercourse and presence of seminal fluid are essential factors for pregnancy. With respect to the latter, studies have shown that the soluble and 
exosome-born signaling agents present in the semen interact with the female reproductive tract and induce an immune response that may benefit the pregnancy outcome [1]. However, whether physical act of sexual intercourse has any beneficial effect on the chance of pregnancy during the cycle of ART is still controversial. Studies have shown that sexual intercourse may cause subclinical upper reproductive tract infection that is associated with decreased implantation rates [2] as well as ascending uterine infection during third-trimester gestation[3]. Indeed, the uterus is very vulnerable to pathogens introduced by sexual intercourse because the cervical mucus barrier to ascending infection is disrupted by mechanical passage of the catheter during the embryo transfer procedure of IVF treatment. Moreover, the act of intercourse may induce the event of orgasm that increases the activity of uterine myometrium [4]. Therefore, the intercourse-induced increases in levels of uterine activity are highly correlated with uterine contractions that may be beneficial for embryo implantation and affect IVF outcome[5].

On the other hand, sexual intercourse has beneficial effects on assisted implantation. Studies performed using animal models revealed that exposure of the female reproductive tract to either seminal plasma or fluid component of the ejaculate is beneficial to achieving successful embryo implantation and normal embryo development [6-8]. For instance, rodent studies performed using artificial insemination or embryo transfer with being exposed to seminal plasma have substantially higher pregnancy rates than those without being exposed [6, 7]. Similarly, rat embryos inseminated with spermatozoa before blastocyst transfer have a higher implantation rate than those without spermatozoa exposure [9]. Additionally, increasing evidence suggests that women who have tissue contact with husband's semen during ART treatment improve their pregnancy outcomes [10-12]). A meta-analysis study performed using seven randomized controlled trials that involved 2,204 patients showed that women with either sexual intercourse or passive exposure to semen around the time of IVF treatment had an increase in clinical pregnancy rate (approximately 23\%) [11]. Collectively, these findings indicate that components in the semen may induce an immune response that may positively affect the endometrial receptivity and pregnancy outcomes.

Interestingly, sexual arousal has been shown to improve the motility of spermatozoa, although the detailed molecular mechanism is not clear [13]. Furthermore, studies revealed that patients with sexual intercourse without contraception had a higher incidence of the risk of life-threatening complication as a heterotopic pregnancy as a consequence of spontaneous pregnancy after sexual intercourse during their frozen-thawed embryo transfer (FET) cycles [14]. At present, it remains unclear whether the beneficial effects of sexual intercourse on the endometrial receptivity and embryo implantation are due to the transportation of semen into the vagina or the sexual behavior itself. Therefore, we aimed to investigate whether the act of sexual intercourse itself (using condoms as contraception) has any influence on clinical pregnancy rates in patients with IVF assisted treatment.

\section{Methods}

\section{Study design}


This is a unblinded randomized study conducted at the Center for Reproduction and Genetics of the Affiliated Hospital of Shandong University of Traditional Chinese Medicine. This study was approved by the University Research Ethics Committee and registered at the China Clinical Trial Registration Center (registration number ChiCTR1800017209). All participants signed an informed consent form prior to the study. The sample size calculation was done based on previous studies, in which the efficacy of sexual intercourse during the peri-transfer period was $24 \%[10], a=0.05$ and $\beta=0.2$. According to the ratio of $1: 1$, $10 \%$ of the lost visit rate, there were expected to be 104 patients in the the barrier contraception group and 104 patients in the abstain group.

\section{Study Participants}

A total of 300 infertile patients undergoing IVF frozen-thawed cycle embryo transfer (FET) were recruited from the Center for Reproduction and Genetics of the Affiliated Hospital of Shandong University of Traditional Chinese Medicine, from 19 July 2018 to 24 February 2019,during this period, patients undergoing embryo transfer will be included after signing informed consent. Inclusion criteria were: 1) at least one cycle of IVF/ICSI performed in our reproductive center; 2) female age $\leq 35$ years; 3 ) a body mass index (BMI) between 18.5 and $25 ; 4)$ regular menstrual cyclicity (24-35 days); 5) the attending physician and embryo transfer being the same person for all patients; 6 ) the tubal factor is the cause of female infertility ( bilateral tubal obstruction, or hydrosalpinx treated using laparoscopic surgery); 7) 4 or more embryos available for transfer with at least one viable day 3 embryo with a score of 6 or above according to the Istanbul Consensus[20] and without receiving preimplantation genetic screening/preimplantation genetic diagnosis (PGS/PGD). Exclusion criteria were 1) patients with known congenital uterine malformation, uterine fibroids, or endometriosis; 2) chromosomal abnormalities in any of infertile couples; 3 ) female with immune diseases. Three hundred patients (all due to tubal factors) requiring IVF treatment were randomly divided into two groups, with 150 patients in each group. Group $A$ $(n=150)$ was subjected to have intercourse using a condom at the night before embryo transfer, while group $B(n=150)$ was subjected to abstain for the entire IVF cycle. Patients engaged in intercourse one night before embryo transfer using a barrier contraception (condoms). All patients participating in the study underwent vaginal scrubbing prior to embryo transfer.

\section{Randomization}

Patients are randomly grouped according to the random list generated by the computer. The exact time of randomization was on the day of endometrial transformation. Randomization via website( www.randomization.com ).The online randomization program was operated by a special data expert, who did not participate in patient recruitment and clinical management. The person then prepares the randomly grouped cards and puts them in an opaque envelope. On the day of randomization, the subjects were randomly divided into two groups according to the opaque envelope. Group $A(n=150)$ was subjected to have intercourse using a condom at the night before embryo transfer, while group $B(n=150)$ was subjected to abstain for the entire IVF cycle. Signed written informed consents were obtained from 
all participants before the study. Information about whether patients engage sexual intercourse using the barrier contraception or to abstain one night before FET is sealed in an opaque envelope with only one number on it. In order to prevent patients from not following the doctor's orders, we will contact the patients and their spouses again according to the results after unblinding to confirm that the content provided is true.

\section{Interventions}

In the present study, artificial endometrium preparation by the exogenous administration of estrogen and progesterone was used for the thawed embryo transfer cycle. To prepare the endometrium, all patients self-administered oral estradiol valerate $8 \mathrm{mg}$ per day started at the third to fourth day of the menstrual period for 5 days, and then $12 \mathrm{mg}$ per day for the remaining days of the cycle, which was adjusted according to the clinical situation during this period. Estrogen administration was continued until the endometrium reaches a thickness of $8 \mathrm{~mm}$ (determined using an ultrasonographic examination). and then progesterone was combined to initiate the secretory phase in preparation for FET. Luteal support was routinely provided after FET for 14 days irrespective of pregnancy. For patients confirmed with a clinical pregnancy,estradiol valerate and progesterone were continued until 10 weeks of gestation, which was gradually reduced until detecting the fetal heart beats.

\section{Participant Follow-up And Data Collection}

Serum $\beta$-hCG levels were examined using electrochemiluminescence analysis (Cobas e411 System Product, Germany) on day 14 following embryo transfer in all patients. Implantation was defined as a serum quantitative $\beta$-hCG $\geq 10 \mathrm{u} / \mathrm{L}$. All pregnancies were followed until 7 weeks after FET. A biochemical losses is defined as a transient but significant increase in serum $\beta$-hCG $\geq 10 \mathrm{u} / \mathrm{L}$ between days 12 and 20 after embryo transfer without detecting a gestational sac by an ultrasonography. A clinical pregnancy is defined as the detection of fetal heart beats using ultrasonographic examinations 35 days after FET. An early abortion is defined as the detection of an empty gestational sac using ultrasonographic examinations before 12 weeks of gestation. Implantation rate is defined as the ration of the number of embryos implanted and the number of transferred embryos.

\section{Statistical analysis}

The statistical software package, IBM SPSS Statistics for Windows, version 22.0 (IBM Corporation, Armonk, NY, USA), was used for all data analyses. The data are expressed as mean \pm standard deviation $(x \pm s)$. Among those were normally distributed and homogeneity of variance (homoscedasticity) was satisfied, a $t$ test was performed; otherwise, Mann-Whitney $\mathrm{U}$ test was performed. The treatment outcomes for each group were counted and expressed as a frequency. When the total sample size was greater than 40 and the minimal theoretical frequency was greater than 5, we applied the Chi-square test. When the total sample size was less than 40 or the minimal theoretical frequency was less than 5 , we 
applied the Fisher exact-probability method. P 0.05 was considered statistically significant. The intention to treat analysis will not be performed because we had only one intervention after randomization and did not use any experimental drugs .

\section{Results}

Owing to non-compliance with medication and personal concerns resulting in cycle cancelation. Ultimately, 223 patients were included in the analysis, with 116 cases in group A and 107 cases in group B (Fig. 1). The characteristics of trial participants were similar, confirming the adequacy of the randomization process. No significant difference in major parameters between the two groups, including maternal age (years old) $(30.27 \pm 3.58 \mathrm{vs}$. $29.83 \pm 3.05)$, body mass index $\left(B M l, k g / m^{2}\right)(22.04 \pm 2.32 \mathrm{vs}$. $22.48 \pm 1.81)$, average FSH concentrations (U/L) $(6.54 \pm 1.79$ vs. $6.94 \pm 1.77)$, duration of infertility (years) $(2.33 \pm 1.40$ vs. $2.64 \pm 1.33)$, endometrial thickness on the day of FET $(\mathrm{mm})(10.50 \pm 1.55$ vs. $10.53 \pm 1.45)$, number of good embryos ( $3.10 \pm 1.35$ vs. $2.79 \pm 1.19)$, number of previous pregnancies $(1.19 \pm 1.26 \mathrm{vs}$. $1.27 \pm 1.36)$, number of births ( $0.41 \pm 0.53$ vs. $0.37 \pm 0.52)$, type of infertility (primary infertility) $(42.24 \%$ [49/116]) vs. $42.99 \%$ [46/107]), and history of spontaneous abortion (20.69\% [24/116) vs. $17.76 \%$ [19/107]) (Table 1).

Notably, a significant difference in the clinical pregnancy rate $(51.72 \%[60 / 116]$ vs. $37.07 \%[86 / 232], P=$ 0.045 ) was present between the two groups (Table 2). Similarly, the implantation rate was significantly higher in group A than in group B (38.31\% [41/107], vs. 24.77\% [53/214], $\mathrm{P}=0.005)$ (Table 2). However, no significant difference in the biochemical losses rate was present between two groups $(11.43 \%$ [8/70] vs. $8.7 \%[4 / 46], P=0.761$ ) (Table 2). Similarly, although few case numbers, there was no significant difference of the spontaneous abortion rate between two groups $(11.67 \%[7 / 60]$ vs. $14.63 \%[6 / 41]), P=$ 0.662) (Table 2).

\section{Discussion}

Assisted reproductive technology (ART) has evolved rapidly over the past 30 years, which is as frequent for conceiving a child as any other minor medical procedure. Despite the recent advances in IVF, the successful rates (clinical pregnancy and subsequent live birth rates) remains disappointingly low because of the multiple causes of infertility, individual patient differences, and intolerance to embryo transfer. Among these multiple factors, the implantation rate is limited despite the transfer of excellentquality embryos. Indeed, repeated failures of implantation due to inadequate uterine receptivity has become a compelling challenge in reproductive medicine. A meta-analysis revealed a statistically significant improvement in clinical pregnancy rate when women are exposed to seminal plasma (unprotected sexual intercourse, intra-vaginal, intracervical, or intrauterine application) around the time of oocyte pick-up or embryo transfer [11]. Studies performed using animal models and human samples suggest an immune-regulatory role for seminal plasma in embryo implantation [11]. But at present, no trial study has investigated whether the "mechanical" act of sexual intercourse before the time of embryo transfer has any influence on implantation and clinical pregnancy. 
The results obtained from this randomized study are the first to provide evidence that the act of sexual intercourse (excluding the effect of semen plasma) during the time of embryo transfer is not detrimental to implantation or pregnancy outcome. Furthermore, female patients having sexual intercourse exhibited substantial improvement in both implantation and pregnancy rates. All patients underwent artificial cycle FET. Therefore, natural conception of the enrolled patients in the sexual intercourse group was unlikely to occur.

The observed findings that sexual intercourse had a beneficial effect on pregnancy outcome may be explained by several factors. The physical act of sexual intercourse arousal has been shown to increase the blood flow of female genital tract [15], which could enhance the blood supply of the endometrium, resulting in improvement of endometrial receptivity. Indeed, increasing evidence suggests that adequate uterine perfusion contributes to the uterine receptivity, and an increased impedance in uterine flow is associated with infertility $[16,17]$. Future studies are required to investigate the distribution of endometrial blood flow and the impedance changes in uterine artery blood flow before and after sexual intercourse. Previous studies also showed that sexual intercourse can activate various female genital tract reflexes, which may further induce several beneficial effects, such as increased production of sex hormones and maintenance of general tract functionality [13]. The other potential beneficial impact of sexual intercourse on the pregnancy outcome is that coitus is considered as a type of behavioral treatment approach that might be efficacious for emotional aspects of infertility. A study performed including 54 infertile patients showed that behavior treatment significantly decreased degree of anxiety, depression, and fatigue, but increased vigor, of which $34 \%$ of these women became pregnant later on [18]. Therefore, if the act of sexual intercourse can make women less stressful, it may, to some extent, reduce the anxiety of patients perceived prior to embryo transfer. Moreover, encouraging couples to engage in sexual intercourse during their IVF treatment gains an additional psychological advantage, as they might feel more natural and comfortable during the process of conception. Certainly, all these proposed mechanisms are issues worth exploring further.

Of course, there are some limitations to this study. For example, it is important to note that our selected time of coitus for patients was only once, the night before the embryo transfer. Our interpretation of the results obtained in the present study has been too brief an interval to show beneficial effects. It is difficult to determine which day is the best time point for sexual intercourse between oocyte pick-up and embryo transfer. However, a study performed using animal models showed that the pregnancy rates after artificial insemination in ewes were much lower than those after natural mating, although the mechanism remains unclear [19]. Therefore, the results obtained from animal studies suggest that the act of natural mating does not appear to increase uterine activity and subsequent pregnancy rate. Future studies aimed at addressing these issues by conducting a study design with various time points of sexual intercourse to investigate the effects on subsequent pregnancy outcomes. Besides, limitations of our study also include the non-blindness and small sample size which will reduce the persuasiveness of our experiments. It also cannot be ignored the fact that the number of good quality embryos transferred in group A has a borderline significance which will also have an impact on the experimental results. 


\section{Conclusions}

In summary, clinical data obtained from this study demonstrate that the "physical" component of sexual intercourse before the day of embryo transfer may improve the clinical pregnancy and implantation rates during FET cycles. However, there is much work to be done in investigating the functional relationships and the underlying mechanisms to support the theoretical basis. A large-sample, multicenter, randomized controlled trial study is therefore needed to confirm the effects of sexual intercourse on IVF outcomes.

\section{Abbreviations}

FET frozen-thawed embryo transfer IVF in vitro fertilization

\section{Declarations}

Ethics approval and consent to participate[This study was approved by the University Research Ethics Committee. All participants signed an informed consent form prior to the study.

Consent for publication: Publication consent was obtained from all authors.

Availability of data and material: Data and material are available with the corresponding author.

Competing interests: All authors declare that they have no competing interests.

Funding: This study was funded by the National Natural Science Foundation of China (81874484). All authors have no conflict of interest.

Authors' contributions: HJW and YLH responsible for data collection and thesis writing,CXL responsible for data curation and supervision, SJY and SZG responsible for the revision of articles and enrollment of patient. All authors have read and approved the manuscript.

Acknowledgements: We thank ACCDON (www.letpub.com) for its linguistic assistance during the preparation of this manuscript. We thank Jing-Yan Song for his help in statistics.

This work was supported by the National Natural Science Fund project (No. 81874484)

Declaration of Conflict of Interest: None.

\section{References}

1. Robertson SA and Sharkey DJ. Seminal fluid and fertility in women. Fertil Steril 2016: 106; 511-519.

2. Fanchin R, Harmas A, Benaoudia F, Lundkvist U, Olivennes F, and Frydman R. Microbial flora of the cervix assessed at the time of embryo transfer adversely affects in vitro fertilization outcome. Fertil Steril 1998: 70; 866-870. 
3. Naeye RL. Coitus and associated amniotic-fluid infections. N Engl J Med 1979: 301; 1198-1200.

4. Fox CA, Wolff HS, and Baker JA. Measurement of intra-vaginal and intra-uterine pressures during human coitus by radio-telemetry. J Reprod Fertil 1970: 22; 243-251.

5. Fanchin R, Righini C, Olivennes F, Taylor S, de Ziegler D, and Frydman R. Uterine contractions at the time of embryo transfer alter pregnancy rates after in-vitro fertilization. Hum Reprod 1998: 13; 19681974.

6. O WS, Chen HQ, and Chow PH. Effects of male accessory sex gland secretions on early embryonic development in the golden hamster. J Reprod Fertil 1988: 84; 341-344.

7. Pang SF, Chow PH, and Wong TM. The role of the seminal vesicles, coagulating glands and prostate glands on the fertility and fecundity of mice. J Reprod Fertil 1979: 56; 129-132.

8. Queen K, Dhabuwala CB, and Pierrepoint CG. The effect of the removal of the various accessory sex glands on the fertility of male rats. J Reprod Fertil 1981: 62; 423-426.

9. Carp HJ, Serr DM, Mashiach S, and Nebel L. Influence of insemination on the implantation of transferred rat blastocysts. Gynecol Obstet Invest 1984: 18; 194-198.

10. Tremellen KP, Valbuena D, Landeras J, Ballesteros A, Martinez J, Mendoza S, Norman RJ, Robertson $\mathrm{SA}$, and Simón $\mathrm{C}$. The effect of intercourse on pregnancy rates during assisted human reproduction. Hum Reprod 2000: 15; 2653-2658.

11. Crawford G, Ray A, Gudi A, Shah A, and Homburg R. The role of seminal plasma for improved outcomes during in vitro fertilization treatment: review of the literature and meta-analysis. Hum Reprod Update 2015: 21; 275-284.

12. Coulam CB and Stern JJ. Effect of seminal plasma on implantation rates. Early Pregnancy 1995: 1; 33-36.

13. Levin RJ. Do women gain anything from coitus apart from pregnancy? Changes in the human female genital tract activated by coitus. J Sex Marital Ther 2003: 29 Suppl 1; 59-69.

14. Aoki Y,Kumakiri J,Itakura A et al. Should sexual intercourse be avoided during the embryo transfer cycle? Life-threatening ruptured heterotopic pregnancy after single thawed embryo transfer: case report and review of the literature.[J] .Clin Exp Obstet Gynecol, 2017, 44: 489-491.

15. Levin RJ and Wylie KR. The g-spot article-some of its limitations. J Sex Med 2012: 9; 1955-1956; author reply 1956-1957.

16. Goswamy RK, Williams G, and Steptoe PC. Decreased uterine perfusion-a cause of infertility. Hum Reprod 1988: 3; 955-959.

17. Kurjak A, Kupesic-Urek S, Schulman $\mathrm{H}$, and Zalud I. Transvaginal color flow Doppler in the assessment of ovarian and uterine blood flow in infertile women. Fertil Steril 1991: 56; 870-873.

18. Domar AD, Seibel MM, and Benson $H$. The mind/body program for infertility: a new behavioral treatment approach for women with infertility. Fertil Steril 1990: 53; 246-249.

19. Raynal $P$ and Houdeau $E$. [Comparison of the uterine reflex activity during artificial insemination and mating in the ewe]. J Gynecol Obstet Biol Reprod (Paris) 2004: 33; 725-733. 
20. Alpha Scientists in Reproductive Medicine and ESHRE Special Interest Group of Embryology,The Istanbul consensus workshop on embryo assessment: proceedings of an expert meeting.[J] .Hum Reprod, 2011, 26: 1270-83

\section{Tables}

Table 1

Clinical characteristics for enrolled patients with frozen-thawed embryo transfer.

\begin{tabular}{|llll|}
\hline Item & Group A & Group B & P \\
\hline No. of cycles $(\mathrm{n})$ & 116 & 107 & - \\
\hline No. of transferred embryos $(\mathrm{n})$ & 232 & 214 & 0.331 \\
\hline Age (years) & $30.27 \pm 3.58$ & $29.83 \pm 3.05$ & 0.116 \\
\hline BMI (kg/m ${ }^{2}$ ) & $22.04 \pm 2.32$ & $22.48 \pm 1.81$ & 0.103 \\
\hline bFSH (IU/L) & $6.54 \pm 1.79$ & $6.94 \pm 1.77$ & 0.103 \\
\hline Length of infertility (years) & $2.33 \pm 1.40$ & $2.64 \pm 1.33$ & 0.883 \\
\hline Endometrial thickness on FET day (mm) & $10.50 \pm 1.55$ & $10.53 \pm 1.45$ & 0.072 \\
\hline No. of high quality embryos (n) & $3.10 \pm 1.35$ & $2.79 \pm 1.19$ & 0.643 \\
\hline No. of pregnancies & $1.19 \pm 1.26$ & $1.27 \pm 1.36$ & 0.572 \\
\hline No. of births & $0.41 \pm 0.53$ & $0.37 \pm 0.52$ & 0.910 \\
\hline Type of infertility (primary infertility) & $42.24 \%(49 / 116)$ & $42.99 \%(46 / 107)$ & 0.579 \\
\hline History of spontaneous abortion & $20.69 \%(24 / 116)$ & $17.76 \%(19 / 107)$ & \\
\hline Group A: patients were engaged to have sexual intercourse. & & & \\
\hline Group B: patients were engaged to abstain & & & \\
\hline $\begin{array}{l}\text { BMl, body mass index; bFSH, basal follicle-stimulating hormone (on day } 2) ; \text { data are expressed as } \\
\text { means } \pm \text { variance (s). }\end{array}$ & & & \\
\hline
\end{tabular}


Table 2

Comparison of pregnancy outcomes between group A (engage in intercourse) and group B (abstain).

\begin{tabular}{|llll|}
\hline Pregnancy outcome & Group A & Group B & P \\
\hline Clinical pregnancy rate & $51.72 \%(60 / 116)$ & $38.31 \%(41 / 107)$ & 0.045 \\
\hline Implantation rate & $37.07 \%(86 / 232)$ & $24.77 \%(53 / 214)$ & 0.005 \\
\hline Biochemical losses rate & $11.43 \%(8 / 70)$ & $8.70 \%(4 / 46)$ & 0.761 \\
\hline Spontaneous abortion rate & $11.67 \%(7 / 60)$ & $14.63 \%(6 / 41)$ & 0.662 \\
\hline
\end{tabular}

Figures 


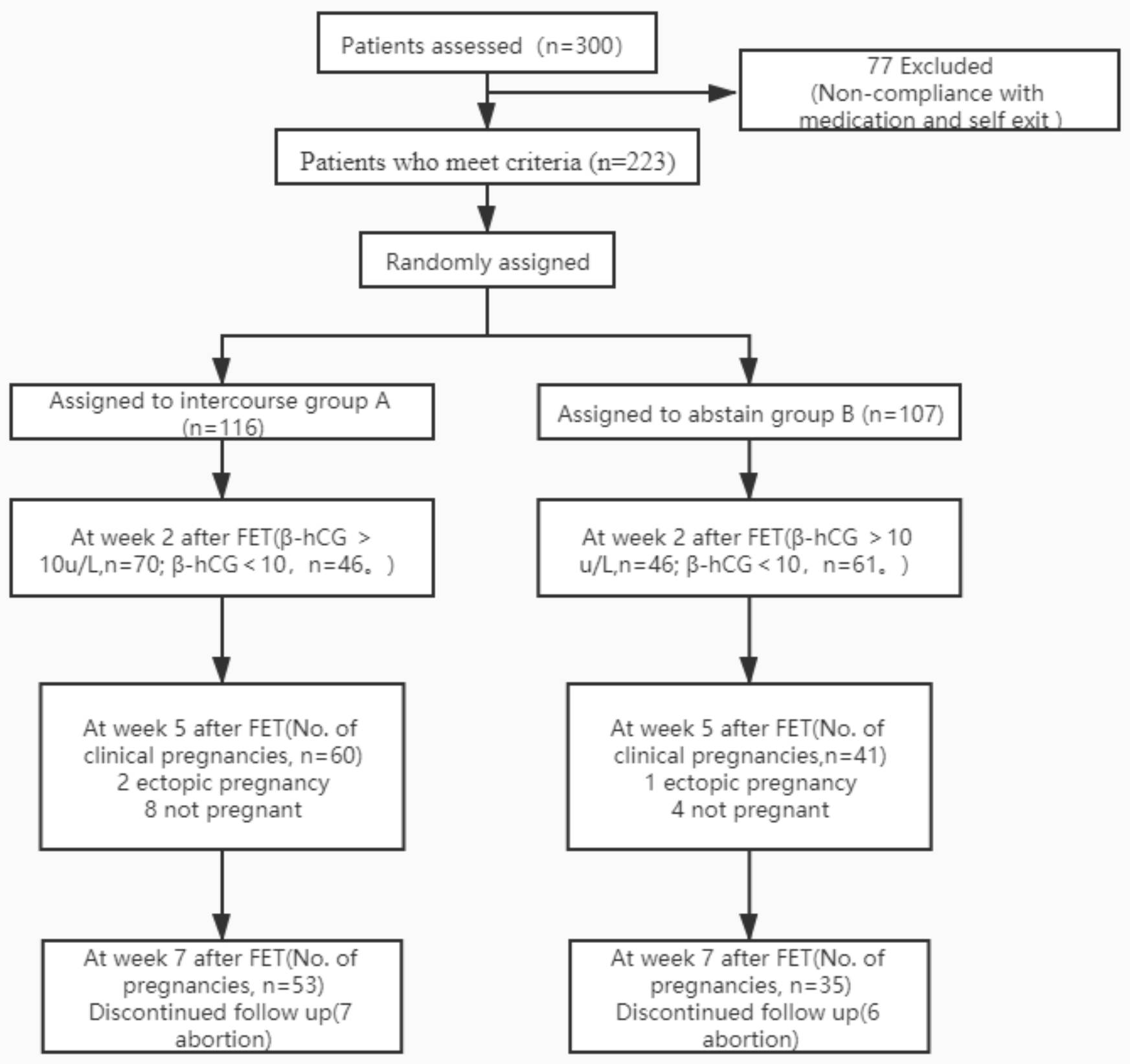

Figure 1

Flow chart of case selection. 